\title{
Associations between Dietary Diversity Scores and Obesity Phenotypes in Women
}

\author{
Golnoosh Goodarzi ${ }^{1}$, Seyyed Ali Keshavarz ${ }^{1}$, Firoozeh Hosseini-Esfahani ${ }^{2}$, Asal Ataie-Jafari ${ }^{{ }^{*}}$ \\ 1- Department of Nutrition, Science and Research Branch, Islamic Azad University, Tehran, Iran. \\ 2- Nutrition and Endocrine Research Center, Research Institute for Endocrine Sciences, Shahid Beheshti University of Medical Sciences, Tehran, Iran.
}

\section{A B S T R A C T}

Background and Objectives: Dietary diversity is a proxy measurement of overall dietary quality. The aim of this study was to assess relationships between dietary diversity score and obesity phenotypes in women, Tehran, Iran.

Materials and Methods: In this cross-sectional study, 197 women aged $\geq 25$ years from Municipal District 7 of Tehran were participated. Food intakes were calculated using semi-quantitative food frequency questionnaires. Dietary diversity score was calculated using healthy food diversity score. Anthropometric parameters and physical activities were measured based on the standard methods. Multivariate logistic regression was used to show possible associations between the dietary diversity scores and the obesity phenotypes.

Results: The mean ( \pm standard deviation) age of the participants was $37.0 \mathrm{y} \pm 7.41$. The mean weight, body mass index, waist circumference, waist-to-hip ratio and waist-to-height ratio were $78.3 \mathrm{~kg} \pm 1.2,27.1 \mathrm{~kg} / \mathrm{m}^{2} \pm 34,92.5 \mathrm{~cm} \pm 14.4$, $0.87 \pm 23.4$ and $0.57 \pm 6.97$; respectively. The mean $( \pm \mathrm{SD})$ of dietary diversity score was $0.27 \pm 16.73$. No relationships were seen between the dietary diversity scores and general obesity, high waist-to-hip ratios and waist-to-height ratios. However, risks of high waist circumferences decreased with increasing dietary diversity score [OR(CI): $0.26(0.09-0.75)$; p-trend $=0.04]$.

Conclusions: In conclusion, inverse relationships were seen between the abdominal obesity and the dietary diversity scores in women in Tehran. Therefore, more various diets are recommended for the prevention of obesity in women.

Keywords: Dietary diversity score; Abdominal obesity; Overweight; women, Iran

\section{Introduction}

Overweight and obesity are described as abnormal or excessive fat accumulations that may affcet human health. Levels of fat accumulation and their distributions in the body or around the waist and trunk (abdominal, central or apple-shaped) or visceral (pear-shaped) include important effects on human health. (1). As the number of infectious diseases decreases globally, non-communicable diseases rapidly increase, most of which are associated with nutrition and lifestyle (2). The increasing prevalence of non-communicable diseases is usually due to obesity and overweight. Obesity is one of the most important threats to human health in 21st Century (3). Overweight and obesity are major risk factors of cardiovascular diseases (CVD), high blood pressure, type-2 diabetes, stroke, gallbladder disease, osteoarthritis, sleep apnea, respiratory problems and types of cancers (e.g. breast, prostate and colon cancers). Obesity is prevalent in children and adults in developing and developed countries (4-6). A meta-analysis of studies between 2000 and 2018 demonstrated that the prevalence of general and abdominal obesities increased in Iran (7). In addition, prevalence of obesity in Iranian women has increased from 16 to $20 \%$ only within three years (1).

The origin of obesity includes genetic and environmental/behavioral factors. Environmental/ behavioral factors play important roles in obesity following imbalances between energy consumption and metabolism. However, it is commonly suggested that obesity occurs only due to overeating or lack of physical activities (8). In addition to important effects of foods on obesity, various combinations of foods and their interactions may be associated with obesity. To study overall diets, dietary diversity score (DDS) can be used. Higher DDS is associated with higher intakes of micro and macronutrients and nutritional adequacy in people. This index is linked to chronic illnesses, metabolic syndromes and heart diseases 
(9). Diets that are high in dietary diversity play protective roles against chronic diseases. Although higher DDS values are associated with higher intakes of fibers, vitamin $\mathrm{C}$ and calcium as the nutrients inversely linked to obesity (10), consumption of more various diets is usually associated with higher energy intakes. Dietary diversity is one of the hallmarks of healthy diets, based on the dietary guidelines of the United States Department of Agriculture (USDA) and the Food Guide Pyramid (11,12). The DDS is a multidimensional US Healthy Food Diversity (HFD) index that, unlike previous indicators, can measure dietary variety, quality and proportionality values based on 2010 Dietary Guidelines for Americans (DGA) (13). Studies have suggested that as dietary diversity increases, the odds of developing obesity also increase (14). In contrast, other studies have found that increases in DDS are associated with decreases in chance of obesity (15) while studies have reported no associations (16). Therefore, there are important questions about obesity and dietary diversity. This cross-sectional study was carried out to investigate relationships between the obesity phenotypes and DDS in women in Tehran.

\section{Materials and Methods}

\section{Study design and participants}

This study was a cross-sectional study carried out in fall and winter, 2018. The sample size was estimated using the following formula:

$\mathrm{C}=0.5 * \ln [(1+\mathrm{r}) /(1-\mathrm{r})]=0.2132$

Total sample size $\mathrm{N}=\left[\left(\mathrm{Z}_{\alpha}+\mathrm{Z}_{\beta}\right) / \mathrm{C}\right]^{2}+3=176$

Where, $\alpha$ was $0.05, \beta$ was 0.2 and correlation between DDS and obesity $(r)$ was set at 0.2 . Based on the formula, the sample size was estimated as 176. After adding nonresponse errors of $15 \%$, an additional 26 individuals were included. Thus, a total of 202 individuals were participated in this study. Furthermore, a random sampling procedure was adapted in this study. Participants included women aged $\geq 25$ years, who referred to the House of Quran (holds Quran programs for kids and adults) and Saraye Mahalleh (institution under direct supervision of the municipality, holding cultural, artistic, recreational, sports or religious classes for the locals) in Municipal District 7 of Tehran. Menopause and pregnant women, those with thyroid disorders and women, who received stimulants or appetite suppressants were excluded from the study. After explaining the study process to the participants, consent forms were signed by them. Then, demographic and physical activity questionnaires were completed.

\section{Dietary assessment}

Usual food intakes by the participants were assessed using valid, reliable, semi-quantitative 168-item food frequency questionnaires (FFQ) (17). The questionnaires were completed by a nutritionist. Participants were asked to report their intakes of each item in day, week, month and year over the past year. The frequency expressed in desired serving for each food item was converted to grams per day (18). Each food or beverage was analyzed using food composition table for the levels of energy and nutrients received by the participants. For mixed foods (e.g. pizzas), foods were calculated based on the sum of ingredients. Due to the limitations of Iranian food component tables, the USDA table was used (19).

\section{Dietary diversity score}

The DDS was developed based on dietary guidelines using 26 food groups and subgroups. To assess quality and diversity in this index, American dietary guidelines were used to create health and weight for each food group. The weighting of each group was based on the proportion of the recommended quantity for each group in a 2000-kcal diet. Weighting of each group is based on the proportion recommended for each group in a 2000-kcal diet, which sums up all groups as 1. Range of the DDS was 0-1 (13). Based on the data from FFQ, the cup of food for each person was calculated. To calculate the food share index, the total food intake was divided by the total proportion. Then, health value (HV) of each food item was achieved using multiplier of the health factor in food shares. Furthermore, HV of each participant's diet was calculated from the total HV index of food groups. In the current study, US healthy food diversity index (US HFD index) based on FFQ data was calculated through the following steps: Total food volume was obtained by sum of overall food volume for each individual, then food shares was defined based on the proportion by volume that each of the twenty-six food groups that represent in each individual's diet; by the multiplying the food share by health factor for each group the health value (HV) of foods were obtained; in order to compute the HV of the diet, the health value of foods for each individual was summed. Eventually, the following equivalent was applied to scale the US HFD index.

$\left(1-\sum\right.$ food shares $\left.{ }_{i}{ }^{2}\right) \times$ health value of the diet

\section{Anthropometric measurements}

Anthropometric data were collected by a nutritionist. Weight was measured with a minimal coverage without shoes, including an accuracy of $100 \mathrm{~g}$ using Xiaomi Scale (Xiaomi, China). Height was measured using tape mounted on the wall without shoes, including an accuracy of $0.5 \mathrm{~cm}$. Waist circumference (WC) was measured in the midpoint between the lower edge of the palpable ribs and the upper edge of the pelvis using resilient tape parallel to the ground. Hip circumference was measured in the most prominent part parallel to the surface. Body mass index (BMI) was calculated by dividing weight in kilograms by 
height in square meters. The general obesity was reported as $\mathrm{BMI} \geq 30 \mathrm{~kg} / \mathrm{m}^{2}(20)$. Abdominal obesity was reported as three indicators of high WC ( $\geq 88 \mathrm{~cm}$ ) (21), high waist to hip ratio (WHR) $(\geq 0.8 \mathrm{~cm})$ and high waist to height ratio (WHtR) ( $\geq 0.05)(22)$.

\section{Covariates}

Demographic data such as age, marital status, number of children and number of family members, job, educational level, ethnicity and smoking status were collected using interviews. Participants' physical activity was assessed using IPAQ-short form questionnaires validated in Iran (23).

\section{Statistical analysis}

Data analyzes were carried out using SPSS software v.21 (SPSS, Chicago, IL, USA) (24) and the significance level was considered as 0.05. Demographic, anthropometric and dietary variables were compared in DDS quartiles using Chi-Square and ANOVA tests. Multivariate logistic regression was used to show associations between the DDS and the obesity phenotypes. Results were reported for crude model, Model 1 (adjusted for age, calorie intake and percent of calories from fat) and Model 3 (additionally adjusted for BMI) for abdominal obesity indices.

\section{Results}

Out of 202 women, data of five women was not included because of incomplete questionnaires. Hence, a total of 197 women completed the study. The mean $( \pm \mathrm{SD})$ age of the participants was $37.0 \mathrm{y} \pm 7.41$ and the mean weight was $78.3 \mathrm{~kg} \pm 1.2$. The mean $( \pm \mathrm{SD})$ of DDS was $0.27 \pm 16.7$. Table 1 shows demographic and anthropometric characteristics of the participants based on the DDS quartiles. Women in DDS quarters did not differ significantly in these variables.

Energy-adjusted dietary intakes are compared between the DDS quartiles groups in Table 2. Women with higher DDS scores included significantly higher fiber, whole and refined grain, vegetable and fruit, soy, legume, discretionary solid fat, added sugar and saturated fatty acid intakes (in the crude model not shown here, egg and chicken intakes were also significant).

Odds ratios and $95 \%$ confidence intervals $(95 \% \mathrm{CI})$ for the association of DDS with general obesity and abdominal obesity are shown in Table 3. No relationships were seen between the DDS and the general obesity, high WHR and WHtR. While the odds of high WC decreased with increasing DDS [OR $(\mathrm{CI})$ : $0.26(0.10-0.70)$; p-trend = 0.03]. After adjustment for covariates, this association was still significant $(\mathrm{p}$-trend $=0.04)$.

Table1. Characteristics of the participants based on the quartiles of the dietary diversity scores

\begin{tabular}{|c|c|c|c|c|c|}
\hline \multicolumn{6}{|l|}{ DDS } \\
\hline & Q1 $(\leq 0.06)$ & Q2 (0.06-0.27) & Q3 (0.27-0.9) & Q4 ( $\geq 0.9)$ & $\mathrm{P}^{*}$ \\
\hline & & $\mathrm{n}=51$ & $\mathrm{n}=52$ & $\mathrm{n}=49$ & \\
\hline Age & $36.7 \pm 1.25$ & $35.0 \pm 0.99$ & $37.3 \pm 1.12$ & $38.0 \pm 1.02$ & 0.16 \\
\hline $\begin{array}{l}\text { Current smoker } \\
\mathrm{n}(\%)\end{array}$ & $12(26)$ & $14(27)$ & $11(21)$ & $11(24)$ & 0.30 \\
\hline $\begin{array}{l}\text { High education level } \\
\mathrm{n}(\%)\end{array}$ & $10(24)$ & $13(25)$ & $13(26)$ & $12(23)$ & 0.56 \\
\hline Physical activity (MET/min/week) & $895 \pm 598$ & $899 \pm 581$ & $927 \pm 633$ & $937 \pm 667$ & 0.31 \\
\hline $\mathrm{WC}(\mathrm{cm})$ & $93.5 \pm 16.5$ & $93.1 \pm 10.4$ & $90.5 \pm 13.0$ & $93.8 \pm 12.9$ & 0.62 \\
\hline $\mathrm{BMI}(\mathrm{Kg} / \mathrm{m} 2)$ & $28.8 \pm 2.26$ & $25.8 \pm 3.5$ & $26.7 \pm 3.9$ & $28.2 \pm 5.6$ & 0.15 \\
\hline WHR & $0.87 \pm 0.13$ & $0.85 \pm 0.06$ & $0.90 \pm 61.2$ & $0.87 \pm 0.11$ & 0.37 \\
\hline WHtR & $0.58 \pm 0.22$ & $0.53 \pm 0.05$ & $0.55 \pm 0.10$ & $0.57 \pm 0.08$ & 0.54 \\
\hline
\end{tabular}


Golnoosh Goodarzi, et al: Dietary diversity scores and obesity

Table 2. Energy-adjusted dietary intakes (mean $\pm \mathrm{SD}$ ) of the participants based on the quartiles of dietary diversity score

\begin{tabular}{llllll}
\hline DDS & \multicolumn{3}{c}{} & & \\
\hline & $\mathrm{Q} 1(\leq 0.06) \mathrm{n}=45$ & $\mathrm{Q} 2(0.06-0.27) \mathrm{n}=51$ & $\mathrm{Q} 3(0.27-0.9) \mathrm{n}=52$ & $\mathrm{Q} 4(\geq 0.9) \mathrm{n}=49$ & $\mathrm{P}^{*}$ \\
\cline { 2 - 6 } Carbohydrate (\%of energy) & $63.9 \pm 1.38$ & $65.6 \pm 1.22$ & $67.1 \pm 1.22$ & $64.3 \pm 1.38$ & 0.26 \\
Protein intake (\%of energy) & $12.8 \pm 0.41$ & $12.3 \pm 0.37$ & $12.7 \pm 0.36$ & $13.2 \pm 0.41$ & 0.43 \\
Total fat (\%of energy) & $27.9 \pm 1.13$ & $25.6 \pm 1.00$ & $27.5 \pm 1.00$ & $25.1 \pm 1.13$ & 0.27 \\
Total fiber intake (g/1000Kcal) & $10.2 \pm 3.11$ & $10.5 \pm 4.09$ & $12.8 \pm 1.34$ & $15.7 \pm 4.51$ & 0.00 \\
Saturated fat (\%of energy) & $8.33 \pm 4.21$ & $7.87 \pm 2.33$ & $7.54 \pm 2.51$ & $7.98 \pm 2.06$ & 0.04 \\
MUFA (\%of energy) & $8.12 \pm 0.43$ & $7.91 \pm 0.38$ & $8.16 \pm 0.38$ & $7.93 \pm 0.43$ & 0.96 \\
PUFA (\%of energy) & $4.57 \pm 0.47$ & $4.32 \pm 0.42$ & $4.67 \pm 0.42$ & $4.65 \pm 0.47$ & 0.94 \\
Whole grains (gr) & $52.8 \pm 0.52$ & $80.0 \pm 0.46$ & $112 \pm 0.46$ & $120 \pm 0.52$ & 0.00 \\
Low-fat milk (cup) & $0.17 \pm 0.00$ & $0.09 \pm 0.00$ & $0.05 \pm 0.00$ & $0.19 \pm 0.00$ & 0.33 \\
Dark green vegetables (cup) & $0.11 \pm 0.21$ & $0.20 \pm 0.19$ & $0.17 \pm 0.25$ & $0.27 \pm 0.22$ & 0.51 \\
Red and orange vegetables (cup) & $0.44 \pm 0.32$ & $0.50 \pm 0.21$ & $0.83 \pm 1.15$ & $0.61 \pm 0.63$ & 0.35 \\
Legumes (cup) & $0.66 \pm 0.23$ & $0.54 \pm 0.21$ & $0.75 \pm 1.52$ & $0.91 \pm 0.47$ & 0.04 \\
Starchy vegetables(cup) & $9.3 \pm 10.0$ & $11.6 \pm 12.9$ & $10.4 \pm 32.6$ & $14.4 \pm 10.3$ & 0.16 \\
Other vegetables(cup) & $0.64 \pm 0.42$ & $0.51 \pm 1.21$ & $0.47 \pm 0.46$ & $0.29 \pm 0.33$ & 0.01 \\
Fruits(cup) & $0.95 \pm 1.50$ & $1.09 \pm 1.00$ & $1.74 \pm 1.43$ & $1.88 \pm 2.41$ & 0.05 \\
Nuts, seeds and soya (gr) & $9.01 \pm 20.6$ & $9.38 \pm 27.5$ & $10.4 \pm 19.7$ & $12.7 \pm 21.31$ & 0.03 \\
Seafood (gr) & $14.8 \pm 0.11$ & $7.36 \pm 21.1$ & $10.9 \pm 13.1$ & $8.11 \pm 52.5$ & 0.72 \\
Oils (gr) & $7.21 \pm 0.27$ & $5.06 \pm 0.50$ & $5.64 \pm 6.35$ & $1.42 \pm 8.06$ & 0.30 \\
Meat (gr) & $17.0 \pm 0.11$ & $9.04 \pm 0.31$ & $19.3 \pm 0.64$ & $21.3 \pm 0.42$ & 0.14 \\
Poultry (gr) & $13.0 \pm 0.41$ & $8.68 \pm 0.13$ & $8.0 \pm 0.81$ & $17.9 \pm 0.59$ & 0.11 \\
Eggs (gr) & $1.47 \pm 3.24$ & $6.10 \pm 4.61$ & $2.16 \pm 20.1$ & $7.51 \pm 11.03$ & 0.28 \\
Refined grains (gr) & $23.5 \pm 0.11$ & $33.0 \pm 0.20$ & $49.7 \pm 0.36$ & $52.7 \pm 0.86$ & 0.00 \\
Discretionary solid fats (gr) & $25.2 \pm 0.13$ & $13.6 \pm 0.09$ & $21.8 \pm 0.56$ & $27.64 \pm 0.46$ & 0.01 \\
Added sugar (gr) & $9.11 \pm 14.8$ & $10.4 \pm 16.4$ & $13.3 \pm 11.4$ & $16.7 \pm 17.7$ & 0.01 \\
\hline Q: Quartiles of the Dietary diversity score. & & & & \\
*ANCOVA test & & & & \\
\#: adjust for total calorie intake & & & & &
\end{tabular}

Table 3. Odds ratios (95\% confidence interval) of obesity and abdominal obesity based on dietary diversity scores

\begin{tabular}{|c|c|c|c|c|c|}
\hline & $\begin{array}{l}\mathrm{Q} 1(\leq 0.06) \\
\mathrm{n}=45\end{array}$ & $\begin{array}{l}\text { Q2 (0.06-0.27) } \\
n=51\end{array}$ & $\begin{array}{l}\text { Q3 (0.27-0.9) } \\
\mathrm{n}=52\end{array}$ & $\begin{array}{l}\text { Q4 ( } \geq 0.9) \\
n=49\end{array}$ & P-trend \\
\hline \multicolumn{6}{|c|}{ Obesity( BMI $\geq 30 \mathrm{~kg} / \mathrm{m} 2)$} \\
\hline Model 1 & 1 & $0.64(0.26-1.59)$ & $0.59(0.25-1.41)$ & $0.51(0.22-1.29)$ & 0.49 \\
\hline Model 2 & 1 & $0.73(0.27-1.97)$ & $0.79(0.29-1.79)$ & $0.57(0.23-1.47)$ & 0.68 \\
\hline \multicolumn{6}{|c|}{ High WHR (WHR $\geq 0.8)$} \\
\hline Model 1 & 1 & $0.79(0.46-6.22)$ & $0.77(0.28-2.09)$ & $0.87(0.24-2.00)$ & 0.50 \\
\hline Model 2 & 1 & $0.84(0.44-7.56)$ & $0.82(0.25-2.64)$ & $0.88(0.22-2.11)$ & 0.52 \\
\hline Model 3 & 1 & $0.95(0.46-6.74)$ & $0.85(0.26-2.74)$ & $0.67(0.22-2.90)$ & 0.46 \\
\hline \multicolumn{6}{|c|}{ High WC ( WC $\geq 0.88)$} \\
\hline Model 1 & 1 & $0.30(0.11-0.82)$ & $0.30(0.11-0.81)$ & $0.26(0.10-0.70)$ & 0.03 \\
\hline Model 2 & 1 & $0.28(0.09-0.86)$ & $0.34(0.11-0.99)$ & $0.26(0.09-0.77)$ & 0.05 \\
\hline Model 3 & 1 & $0.26(0.08-0.81)$ & $0.32(0.11-0.95)$ & $0.26(0.09-0.75)$ & 0.04 \\
\hline \multicolumn{6}{|c|}{ High WHtR ( WHtR $\geq 0.5$ ) } \\
\hline Model 1 & 1 & $0.71(0.17-2.83)$ & $0.28(0.08-0.96)$ & $0.48(0.13-1.74)$ & 0.16 \\
\hline Model 2 & 1 & $0.83(0.19-3.74)$ & $0.35(0.09-1.26)$ & $0.57(0.15-1.56)$ & 0.31 \\
\hline Model 3 & 1 & $0.80(0.18-3.58)$ & $0.34(0.09-1.12)$ & $0.56(0.15-2.19)$ & 0.29 \\
\hline
\end{tabular}

Model 1: Crude model

Model 2: Adjusted for age, total calorie intake and calorie intake from fat

Additional adjustment for BMI Model 3:

Abbreviations: Q: Quartiles of the Dietary diversity index; BMI: body mass index; WC: waist circumference; WHR: waist-to-hip ratio; WHtR: waist-toheight ratio.

\section{Discussion}

The present study was carried out on women over 25 years-old in Tehran, Iran. No associations were found between DDS and general obesity. However, inverse relationships were found between the scores of dietary diversity and WC. Based on the previous studies, DDS is a good indicator of assessing nutrients adequacy and dietary quality as well as relationships between diets and diseases
(25-27). Dietary diversity is an overview of an individual's diet and an indicator for evaluating the overall diet $(28,29)$. In this study, no associations were seen between the DDS and general obesity in this study. Similar studies have reported inverse (30), direct (31) or no associations (32) between DDS and BMI. In this study, inverse associations were reported between the abdominal obesity (high WC) 
and high DDS, similar to those of previous studies (30). However, no associations were seen between DDS, general obesity and WHR (32). Jiavarina et al. found that Sri Lankan adults in higher quartiles of DDS consumed higher quantities of foods, which might lead to higher energy intakes and hence obesity (10). Azadbakht et al. showed that adults in Tehran with higher DDS received more calories and were obese $(33,34)$. Karim Beigi et al. found that adults, who had more various diets, received more calories from breads, cereals, fruits and vegetables and were overweight (35). Since the present study did not show links between the general obesity and DDS, it is concluded that increasing DDS is not always associated with weight gain. This is because increasing consumption of healthy foods such as vegetables, whole grains and fruits increases this index. In fact, a wide variety of vegetables and fruits are available in Iran, but varieties of meat products and refined grains are limited. Previous studies on the dietary diversity in Iran have shown that fruit and vegetable group includes the highest score of food diversity $(36,37)$. Therefore, increases in DDS in Iranian people is majorly due to the consumption of fruits and vegetables. In this study, women with higher DDS consumed more fruits, vegetables, and whole grains, which might be associated with a lower abdominal obesity. Previous studies in the country have shown that higher consumptions of fruits, vegetables (38) and whole grains (39) are well associated with metabolic syndrome (of which abdominal obesity is a component).

Although DDS is based on food guide pyramid, which includes no controls over the energy intakes, people at the highest levels of DDS were at lower risks of increased WC. This might be due to their special choices to increase their food diversity scores. Thus, dietary diversity increased with the selection of more various foods from the fruit and vegetable group. Similar to these results, Azadbakht et al. showed direct relationships between the increased energy intakes and DDS; however, this increase was due to higher consumptions of fruits, vegetables and whole grains, followed by increased intakes of fibers, calcium and vitamin $C$, all were inversely linked to obesity $(40,41)$. One of the strengths of this study is the consideration of potential confounding factors. Cross-sectional design of the present study is one of the limitations. Therefore, the researchers are not be able to interpret results of this study in the form of causal relationships. Thus, prospective studies are needed to verify these relationships. Although it has been tried to control for known confounders, effects of unknown variables cannot be ignored. Findings of the present study have shown inverse relationships between the abdominal obesity (high WC) and DDS. Therefore, it is necessary to further study dietary diversity of the people, especially the variety of fruits and vegetables in diets as significant components.

\section{Financial disclosure}

The authors declare no conflict of interests.

\section{Funding/Support}

No funds were received for this study.

\section{References}

1- Azizi F, Azadbakht L, Mirmiran P. Trends in overweight, obesity and central fat accumulation among Tehranian adults between 1998-1999 and 2001-2002: Tehran Lipid and Glucose Study. Ann Nutr Metab 2005;49:3-8.

2- Ogden CL, Carroll MD, Curtin LR, McDowell MA, Tabak CJ, Flegal KM. Prevalence of overweight and obesity in the United States, 1999-2004. Jama 2006;295(13):1549-55

3- Kelishadi R, Safiri S, Djalalinia S, Miranzadeh S, Motlagh ME, Asayesh $\mathrm{H}$, et al. Health-Related Quality of Life according to the Socioeconomic Status of Living Areas in Iranian Children and Adolescents: Weight Disorders Survey. Iran J Med Sci 2019;44(1):18-27.

4- Wei M, Gaskill SP, Haffner SM, Stern MP. Waist circumference as the best predictor of non-insulin- dependent diabetes mellitus compared to BMI, WHR other anthropometric measurements in Mexican Americans: a 7year prospective study. Obes Res 1997; 5:16-23.

5- Popkin BM, Adair LS, Ng SW. Global nutrition transition and the pandemic of obesity in developing countries. Nutr reviews 2012;70(1):3-21.

6- Kelishadi R. Childhood overweight, obesity, and the metabolic syndrome in developing countries. Epidemiol reviews. 2007;29(1):62-76.

7- Vaisi-Raygani, A., Mohammadi, M., Jalali, R. et al. The prevalence of obesity in older adults in Iran: a systematic review and meta-analysis. BMC Geriatr 19, 371 (2019).

8- Serra-Majem L, Bautista-Castano I. Etiology of obesity: two "key issues" and other emerging factors. Nutr Hosp. 2013;28 Suppl 5:32-43.

9- Hedley AA, Ogden CL, Johnson CL, Carroll MD, Curtin LR, Flegal KM. Prevalence of overweight and obesity among US children, adolescents, and adults, 1999-2002. Jama 2004;291(23):2847-50.

10- Jayawardena R, Byrne NM, Soares MJ, Katulanda P, Yadav B, Hills AP. High dietary diversity is associated with obesity in Sri Lankan adults: an evaluation of three dietary scores. BMC public health. 2013;13(1):314.

11- Hatloy A, Torheim LE, Oshaug A. Food variety - a good indicator of nutritional adequacy of the diet? A case study from an urban area in Mali, West Africa. Eur J Clin Nutr 1998 Dec; 52(12): 891-8 22.

12- Kennedy ET, Ohls J, Carlson S, Fleming K. The Healthy Eating Index: design and applications. J Am Diet Assoc 1995 Oct; 95(10): 1103-8.

13 - Vadiveloo M, Dixon LB, Mijanovich T, Elbel B, Parekh N. Development and evaluation of the US Healthy Food Diversity index. Br J Nutr 2014;112(9):1562-74.

14-Karimbeiki R, Pourmasoumi M, Feizi A, Abbasi B, Hadi A, Rafie N, et al. Higher dietary diversity score is associated with obesity: a case-control study. Public health 2018;157:127-34. 
15. Azadbakht L, Esmaillzadeh A. Dietary diversity score is related to obesity and abdominal adiposity among Iranian female youth. Public Health Nutr. 2011;14(1):62-9.

16. Azadbakht L, Zaribaf F, Haghighatdoost F, Esmaillzadeh A. Association of dietary diversity score with obesity and central adiposity among female university students in Isfahan, Iran. Iran J Nutr Sci Food Technol 2010; 5 (2) :27-34.

17- Mirmiran P, Esfahani FH, Mehrabi Y, Hedayati M, Azizi F. Reliability and relative validity of an FFQ for nutrients in the Tehran lipid and glucose study. Public Health Nutr 2010;13:654-62.

18- Food composition table (FCT). Food and Nutrition Information Center, US Department of Agriculture, www.nal.usda.gov/fnic/foodcomp,2010.

19-Azar M. Food Composition Table of Iran. Tehran: National Nutrition and Food Research Institute UeS.

20-Ghaffarpour M, Houshiar-Rad A, Kianfar H. The manual for household measures, cooking yields factors and edible portion of foods. Tehran: Keshaverzi Press, 1999 [In Persian].

21- National Cholesterol Education Program (NCEP). Expert panel on detection, evaluation, and treatment of high blood cholesterol in adults: Adult Treatment Panel III. Circulation 2002;106: 3143-421

22- Moslehi N, Ehsani B, Mirmiran P, Hojjat P, Azizi F. Association of dietary proportions of macronutrients with visceral adiposity index: non-substitution and iso-energetic substitution models in a prospective study. Nutrients 2015;7(10):8859-70

23- Moghaddam, M. H. B., Aghdam, F. B., Jafarabadi, M. A., Allahverdipour, H., Nikookhelat, S. D., \& Safarpour, S. (2018). The Iranian Version of IPAQ in Iran: Content and Construct Validity, Factor Structure, Internal Consistency and Stability. World Appl Sci J 18(8), 1073-1080.

24- Spss I. IBM SPSS statistics version 21. Boston, Mass: International Business Machines Corp. 2012;126.

25-Drewnowski A, Renderson SA, Driscoll A, Rolls BJ. The Dietary Variety Score: assessing diet quality in healthy young and older adults. J Am Diet Assoc 1997; 97: 266-71.

26-Azadbakht L, Mirmiran P, Azizi F. Variety scores of food groups contribute to the specific nutrient adequacy in Tehranian men. Eur J Clin Nutr 2005; 59: 1233-40

27-Poorrezaeian M, Siassi F, Qorbani M, Karimi J, Koohdani F, Asayesh $\mathrm{H}$, et al. Association of dietary diversity score with anxiety in women. Psychiatry Res 2015; 230: 622-7.

28- Wahlqvist ML, Lo CS, Myers KA. Food variety is associated with less macrovascular disease in those with type II diabetes and their healthy controls. J Am Coll Nutr 1989;8(6):515-23.
29- Azadbakht L, Zaribaf F, Haghighatdoost F, Esmaillzadeh A Association of dietary diversity score with obesity and central adiposity among female university students in Isfahan, Iran. Iran J Nutr Sci Food Technol 2010;5(2):27-34.

30-Azadbakht L, Esmaillzadeh A. Dietary diversity score is related to obesity and abdominal adiposity among Iranian female youth. Public Health Nutr 2011; 14: 62-9.

31-Azadbakht L, Mirmiran P, Azizi F. Dietary diversity score is favorably associated with the metabolic syndrome in Tehranian adults. Int J Obes 2005; 29: 1361-7.

32-Ajani S. An assessment of dietary diversity in six Nigerian States. Afr J Biomed Res 2010; 13: 161-7.

33- Azadbakht L, Mirmiran P, Esmaillzadeh A, Azizi F. Dietary diversity score and cardiovascular risk factors in Tehranian adults. Public Health Nutr 2006; 9: 728-36.

34- Mirmiran P, Azadbakht L, Esmaillzadeh A, Azizi F. Dietary diversity score in adolescents-a good indicator of the nutritional adequacy of diets: Tehran lipid and glucose study. Asia Pac J Clin Nutr 2004; 13: 56-60.

35-. Karimbeiki R, Pourmasoumi M, Feizi A, Abbasi B, Hadi A Rafie $\mathrm{N}$, et al. Higher dietary diversity score is associated with obesity: a case-control study. Public Health 2018;157:127-34.

36- Mirmiran P, Azadbakht L, Azizi F. Dietary diversity within food groups: an indicator of specific nutrient adequacy in Tehranian women. J Am Coll Nutr 2006; 25(4):354-61.

37- Steyn N, Nel J, Nantel G, Kennedy G, Labradarios D. Food variety and dietary diversity scores; are they good indicators of dietary adequacy? Public Health Nutr 2006;9:644-50.

38- Esmaillzadeh A, Kimiagar M, Mehrabi Y, Azadbakht L, Hu FB, Willett WC. Fruit and vegetable intakes, C-reactive protein and the metabolic syndrome. Am J Clin Nutr 2006; 84: 1489-97.

39-. Esmaillzadeh A, Mirmiran P, Azizi F. Whole-grain consumption and the metabolic syndrome: a favorable association in Tehranian adults. Eur J Clin Nutr 2005;59:35362.

40- Mirmiran P, Hosseini Esfahani F, Mehrabi Y, Hedayati M, Azizi F. Reliability and relative validity of an FFQ for nutrients in the Tehran Lipid and Glucose Study. Public Health Nutr 2010; 13: 654-62.

41- Kant AK, Graubard BI. A comparison of three dietary pattern indexes for predicting biomarkers of diet and disease. $\mathrm{J}$ Am Coll Nutr 2005; 24: 294-303. 